\title{
High-aspect 3D two-photon polymerization structuring with widened objective working range (WOW-2PP)
}

\author{
Kotaro Obata, Ayman El-Tamer, Lothar Koch, Ulf Hinze and Boris N Chichkov \\ We developed a novel two-photon polymerization (2PP) configuration for fabrication of high-aspect three-dimensional (3D) structures, \\ with an overall height larger than working distance of the microscope objective used for laser beam focusing into a photosensitive \\ material. This method is based on a modified optical 2PP setup, where a microscope objective (100× high N.A.), immersion oil and \\ cover glass can be moved together into the photosensitive material, resulting in an effective higher and wider objective working range \\ (WOW-2PP). The proposed technique enables the fabrication of high-aspect structures with sub-micrometer process resolution. 3D \\ structures with a height of $7 \mathrm{~mm}$ are demonstrated, which could hardly be built with the conventional 2PP set-up due to refractive index \\ mismatch and laser beam disturbances.
}

Light: Science \& Applications (2013) 2, e116; doi:10.1038/lsa.2013.72; published online 6 December 2013

Keywords: femtosecond laser; large-scale structuring; laser material processing; photochemistry; two-photon polymerization

\section{INTRODUCTION}

With ultrafast laser systems, high-intensity laser pulses in a localized focal volume and time can be generated. This allows for novel laser applications in material processing based on nonlinear laser-matter interactions. $^{1-6}$ An attractive three-dimensional (3D) micro- and nanofabrication method using ultrafast laser pulses is the two-photon polymerization (2PP) technique of photosensitive materials. ${ }^{7-15}$ In the 2PP process, femtosecond (fs) laser pulses initiate 2PP via two-photon absorption and subsequent polymerization in an extremely localized focal volume. This results in 3D micro- and nanoscale structures. The minimum resolution of the polymerized structures can be much smaller than the diffraction limit of the incident laser beam. So far, the $2 \mathrm{PP}$ process was primarily optimized for better process resolution, which is widely studied and reported. ${ }^{16-20}$ At the same time, large-scale $2 \mathrm{PP}$ structuring to build structures with centimeter sizes and submicron resolution is required for many applications. However, in the conventional $2 \mathrm{PP}$ process, the structure height is limited by the working distance of the microscope objective used for focusing laser pulses into the photosensitive material. A typical working distance of a $100 \times$ oil immersion type microscope objective is less than $170 \mu \mathrm{m}$. Bückmann et $a l^{21}$ suggested dipping the microscope objective directly into a special photoresist material. However, this method can only be applied with a limited number of photosensitive materials. Commercially available oil immersion-type microscope objectives are not designed for direct use with the photoresist material instead of the immersion oil. Alternatively, a lower-magnification microscope objective lens (e.g., 10×) with much larger working distance can be used for the fabrication of larger structures. However, this is done at a cost of worse process resolution. Thus, often a tradeoff has to be made between working distance of the microscope objective and process resolution.
In this paper, we present a novel approach for the $2 \mathrm{PP}$ structuring, which maintains a classical optical configuration of objective, immersion oil and cover glass, but which allows moving them together dynamically and arbitrarily through the photosensitive medium. This results in a 2PP system with a higher and wider objective working range (WOW-2PP). In addition, fabrication of structures with up to $7 \mathrm{~mm}$ height using a lower magnification microscope objective $(10 \times)$ is demonstrated.

\section{MATERIALS AND METHODS}

Figure 1a shows a schematic illustration of the experimental setup for WOW-2PP. In this experiment, a high-repetition rate Ti:Sapphire fs laser system (Chameleon XR; Coherent $\mathrm{GmbH}$, Diesburg, Germany; repetition rate: $80 \mathrm{MHz}$, wavelength: $780 \mathrm{~nm}$, pulse width: $<140 \mathrm{fs}$ ) is used. The laser power is precisely adjusted by a combination of $\lambda / 2$ wave-plate and polarizing beam splitter cube. The photo-polymerized structure is generated layer-by-layer with a combination of an $x-y$ galvanometric mirror scanner (Hurry SCAN 14, SCANLAB AG, Munich, Germany) and a high-resolution linear stage in $\mathrm{Z}$-axis (M-126.DG1; Physik Instrumente $\mathrm{GmbH} \& \mathrm{Co}$. KG, Karlsruhe, Germany). The laser beam is focused into the sample with a microscope objective. For online monitoring of the $2 \mathrm{PP}$ process, a CCD camera is mounted behind a dielectric mirror placed in front of the $x-y$ galvanometric mirror scanner. The polymerized structure can be monitored due to changes in the refractive index of photosensitive material induced by the polymerization process.

The unique configuration of the WOW-2PP technique with highand low- magnification microscope objectives is illustrated in Figure $1 \mathrm{~b}$ and $1 \mathrm{c}$, respectively. In the case of a high-magnification lens (Figure 1b), a $170 \mu \mathrm{m}$ thick cover glass is fixed below the $100 \times$ oil immersion microscope objective (Plan Apochromat, N.A.=1.40; 


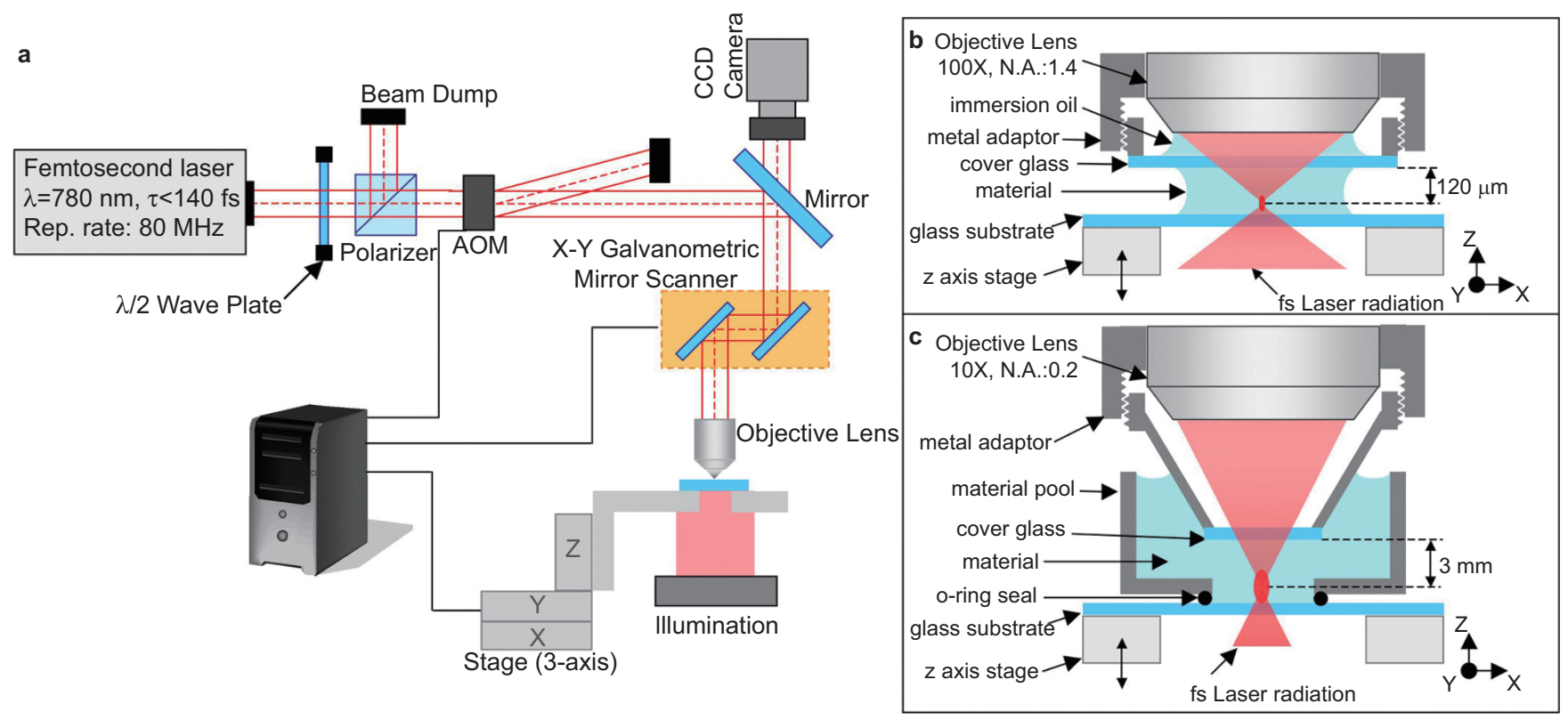

Figure 1 (a) Schematic illustration of experimental setup. (b) Configuration of WOW-2PP with 100x high-magnification microscope objective. (c) Configuration of $10 \times$ low-magnification microscope objective. 2PP, two-photon polymerization.

Zeiss, Göttingen, Germany) at a constant distance with a metal adaptor. This adaptor consists of two parts that are connected with a fine-thread screw with a pitch of $0.5 \mathrm{~mm}$. When the laser beam focus is set at the glass surface, fluorescence can be observed by the process monitoring camera. By observation of this fluorescence and by adjustment of the fine screw, the focal point of the laser beam can be set $120 \mu \mathrm{m}$ below the lower surface of the cover glass in air. The gap between the microscope objective lens and the upper surface of the cover glass is filled with the refractive index matching oil $\left(n_{\mathrm{oil}}=1.515\right)$. A droplet of liquid photosensitive material is put between the cover glass and glass substrate located at a distance of $120 \mu \mathrm{m}$. Thus, the combination of microscope objective and cover glass can be moved together arbitrary in the liquid photoresist, and this movement is not limited by the working distance of the microscope objective used for the $2 \mathrm{PP}$ structuring. In the case of the low-magnification lens, a $10 \times$ microscope objective (Epiplan N.A. $=0.2$; Zeiss) is used as shown in Figure 1c. The microscope objective and the cover glass are also fixed with a metal adaptor. The focal point of the laser beam is set $3 \mathrm{~mm}$ below the lower surface of the cover glass using the same method as mentioned above. The gap between the microscope objective and cover glass is filled with air. During the $2 \mathrm{PP}$ process, the sealed microscope objective is dipped into a tray filled with a liquid photosensitive material. The depth of the material pool used in this contribution is $15 \mathrm{~mm}$.

\section{RESULTS AND DISCUSSION}

WOW-2PP can produce structures with an overall height far beyond the working distance of the microscope objective used for laser beam focusing. Figure 2a shows a scanning electron microscope (SEM) image of $6 \times 6$ grid structure fabricated by WOW-2PP with a $100 \times$ microscope objective. The pentaerythritol tetraacrylate containing 1.5 wt- $\%$ of $4,4^{\prime}$-bis (diethylamino) benzophenone photoinitiator is used as a photosensitive material. The laser beam at $33 \mathrm{~mW}$ power is scanned at $4 \mathrm{~mm} \mathrm{~s}^{-1}$ speed. The $z$-axis is moved stepwise by $0.5 \mu \mathrm{m}$ to build $3 \mathrm{D}$ structures layer by layer. After the polymerization process, non-irradiated pentaerythritol tetraacrylate is removed by 2-propanol and dried with a critical point dryer (E3100 Critical Point Dryers; Quorum Technologies Ltd, East Sussex, United Kingdom).
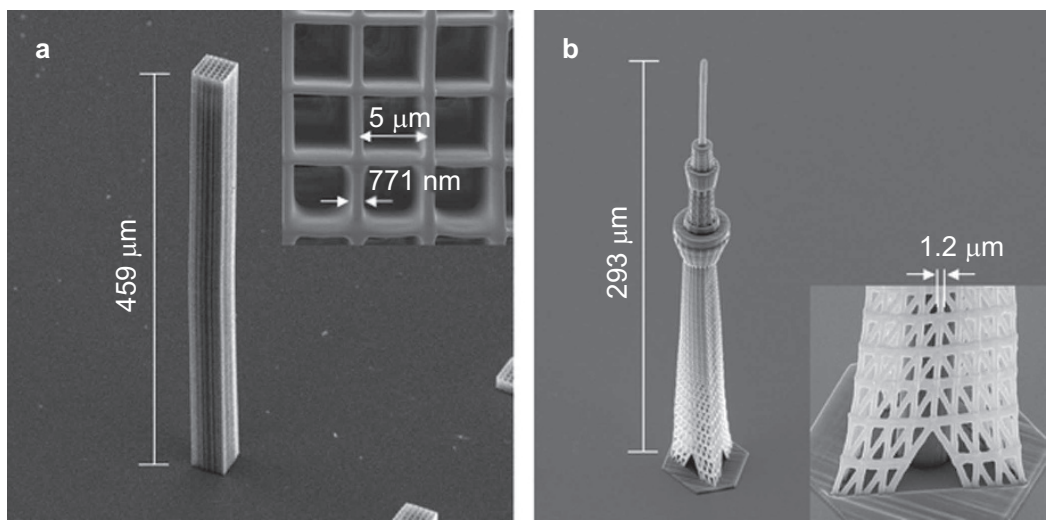

Figure 2 (a) SEM image of $6 \times 6$ grid structure. (b) SEM image of "Tokyo Skytree". Both structured are fabricated by WOW-2PP with $100 \times$ high-magnification microscope objective. 2PP, two-photon polymerization; SEM, scanning electron microscope. 
The SEM image in Figure 2a shows a 3D structure with an overall height of $459 \mu \mathrm{m}$, which is far beyond the working distance of $170 \mu \mathrm{m}$ of the microscope objective. The smallest lateral feature size in this structure is $771 \mathrm{~nm}$. Even at the top of this high-aspect structure, the grid is still regular with a periodic pitch of $5 \mu \mathrm{m}$, without any distortion and shrinkage. Note that this accurate structuring works, although there is a slight flow of photosensitive material, which happens after each layer is finished and the objective is lifted to the next $z$-position. Figure $2 \mathrm{~b}$ shows a microscale television tower ('Tokyo skytree') fabricated at the same conditions as in Figure 2a. The polymerized structure is generated by a combination of an $x-y$ galvanometric mirror scanner and $z$-axis controlled with CAD data. The input laser power and scanning speed are set at $14 \mathrm{~mW}$ and $0.4 \mathrm{~mm} \mathrm{~s}^{-1}$, respectively. The hatching size for laser scanning in the $x-y$ layer is $0.2 \mu \mathrm{m}$; the step of the $z$-axis is $0.5 \mu \mathrm{m}$ at each layer. The SEM image in Figure $2 \mathrm{~b}$ shows an overall height of the tower of $293 \mu \mathrm{m}$, which is also beyond the working distance of $170 \mu \mathrm{m}$ of the microscope objective. The smallest lateral feature size in this structure is $1.2 \mu \mathrm{m}$ and is determined rather by the $\mathrm{CAD}$ data than material characteristics and experimental conditions. Thus, the WOW-2PP technique using high-magnification microscope objective has greater potential for high-resolution large-scale 3D structuring than conventional 2PP.
Furthermore, application of the WOW-2PP technique with lowermagnification microscope objectives has also many advantages. Figure 3 a shows schematic configurations of various types of tube structures with straight, funnel and hourglass shapes. The wall thickness of each tube is designed to be in the range of 50-100 $\mu \mathrm{m}$. All structures are connected to the glass substrate by small feet for removal of the non-irradiated photosensitive material after the 2PP process. Figure $3 \mathrm{~b}$ shows tube structures fabricated with WOW-2PP using a $10 \times$ microscope objective and designs shown in Figure 3a. The nontrombogenic polyethylene glycol diacrylate (SR610; SARTOMER, Colombes, France) with 2 wt-\% IRGACURE 369 (Chiba, Basel, Germany) photoinitiator is used as a photosensitive material. The laser beam is scanned at $150 \mathrm{~mm} \mathrm{~s}^{-1}$. The hatching size in the $x-y$ plane is $2 \mu \mathrm{m}$. The $z$-step between two successive layers is $10 \mu \mathrm{m}$. After the polymerization process, non-irradiated polyethylene glycol diacrylate is removed by ethanol and dried with critical point dryer. The overall height of the generated structures is $7 \mathrm{~mm}$. The hourglass shape structure takes the minimum fabrication time for approximately $90 \mathrm{~min}$. The resulted structure shape corresponds well to the original design, since the air gap between the microscope objective and cover glass can be kept fixed during the whole process. Consequently,
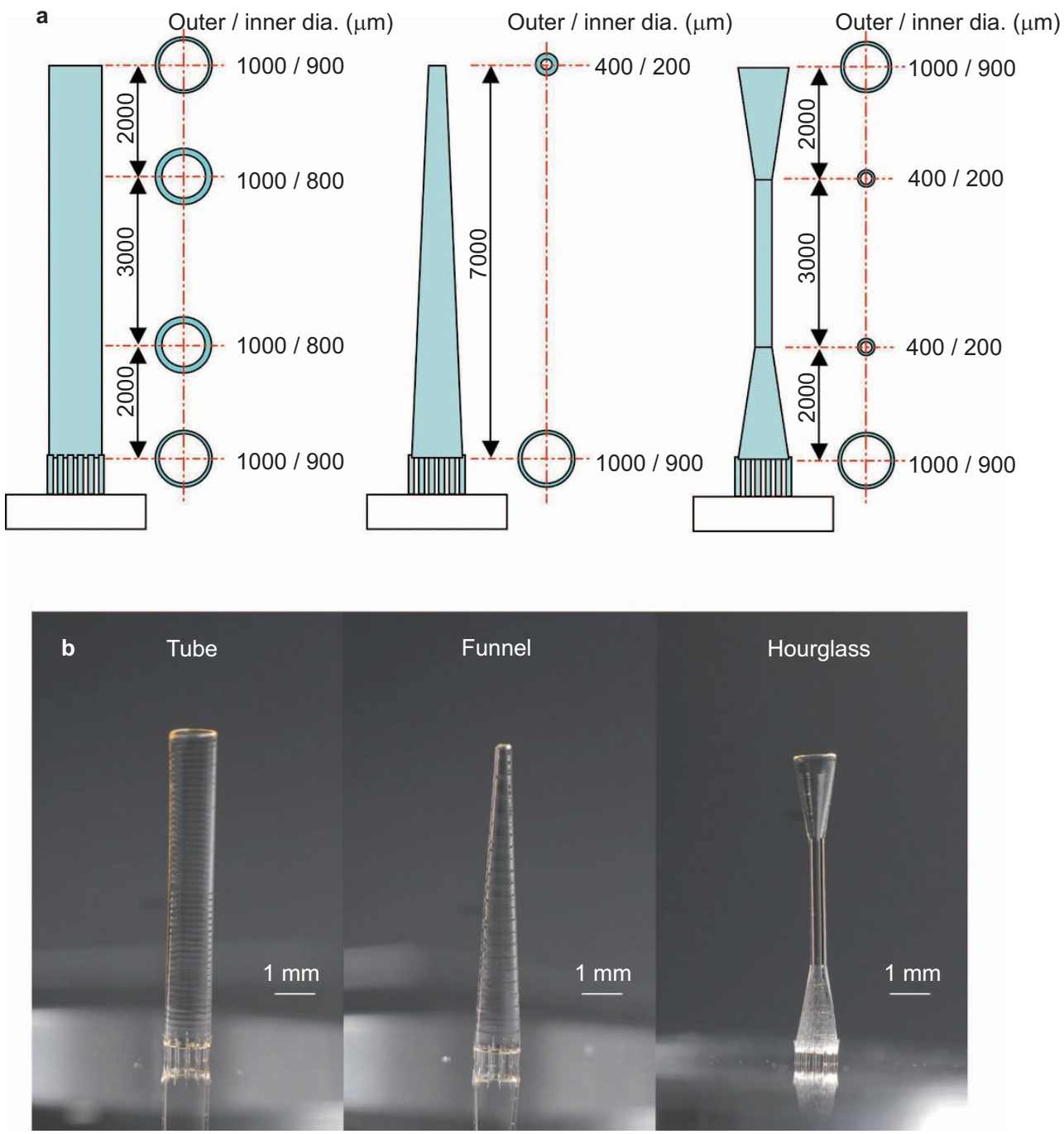

Figure 3 Photograph showing various shapes of tube structure fabricated by WOW-2PP with 10× low-magnification microscope objective. 2PP, two-photon polymerization. 
there is no influence of the beam refraction at the interface between the cover glass and material. The scanning speed of the laser beam used in Figure 3 is $150 \mathrm{~mm} \mathrm{~s}^{-1}$. The scanning speed of the laser beam is a parameter of particular importance for reduction of the processing time. However, only suitable combinations of photopolymer and photoinitiator can make high-speed $2 \mathrm{PP}$ possible. In addition, the laser pulse energy and the repetition rate also influence the morphology of the 2PP structures such as continuity and smoothness of the fabricated lines. In our setup, the applied $x-y$ galvanometric mirror scanners provide very stable laser scanning in the liquid photosensitive material during the $2 \mathrm{PP}$ structuring, resulting in realization of not only large-scale structures, but also high-speed $2 \mathrm{PP}$ processing.

For the demonstration of functionality of the fabricated tubes, a liquid flow test was carried out with the hourglass shape tube shown in Figure 3. The tube was connected at one side to a motorized microsyringe which injected test fluids into the tube. The opposite side was left open as a drain. The test fluid successfully passed the tube; flow rates from 0.1 to $5.0 \mu \mathrm{s} \mathrm{s}^{-1}$ were observed. When 2-propanol was injected into the tube, no clogging or leakage was found. The same results were obtained with Dulbecco's modified Eagle medium: nutrient mixture F-12 (DMEM/ F12; Lonza Ltd, Basel, Switzerland) supplemented with $10 \%$ fetal calf serum and antibiotics. The polyethylene glycol diacrylate is a biodegradable polymer material. Thus, the tube structures fabricated by the WOW-2PP technique can be applied as artificial blood channels.

\section{CONCLUSION}

A novel configuration for the $2 \mathrm{PP}$ fabrication of high-aspect ratio $3 \mathrm{D}$ structures using microscope objective ( $100 \times$ high N.A.), immersion oil and cover glass, which can be moved together through the photosensitive material, has been developed. This technique (WOW-2PP) considerably extends the objective working range providing possibilities for large-scale 3D structuring without laser beam distortions. This technique can be used with all liquid type photosensitive materials. Fabrication of high-aspect ratio structures, impossible with the conventional $2 \mathrm{PP}$ technique, has been demonstrated. For reduction of the processing time, WOW-2PP can be combined with multi-focus structuring. ${ }^{22,23}$ We hope that the demonstrated WOW-2PP technique can bring $2 \mathrm{PP}$ closer to applications.

\section{ACKNOWLEDGMENTS}

This work was supported by the Deutsche Forschungsgemeinschaft (German Research Foundation) Cluster of Excellence REBIRTH and EU/FP7 project Phocam. We thank E Fadeeva and J Koch for helpful technical discussions.

1 Du D, Liu X, Korn G, Mourou G. Laser-induced breakdown by impact ionization in $\mathrm{SiO}_{2}$ with pulse widths from 7 ns to 150 fs. Appl Phys Lett 1994; 64: 3071-3073.
2 Kumagai H, Midorikawa K, Toyoda K, Nakamura S, Okamoto T et al. Ablation of polymer films by a femtosecond high-peak-power Ti:sapphire laser at $798 \mathrm{~nm}$. Appl Phys Lett 1994; 65: 1850-1852.

3 Chichkov BN, Momma C, Nolte S, Alvensleben F, Tünnermann A. Femtosecond, picosecond and nanosecond laser ablation of solids. Appl Phys A 1996; 63: 109115.

4 Davis KM, Miura K, Sugimoto N, Hirao K. Writing waveguides in glass with a femtosecond laser. Opt Lett 1996; 21: 1729-1731.

5 Glezer EN, Milosavljevic M, Juang L, Finlay RJ, Her TH et al. Three-dimensional optical storage inside transparent materials. Opt Lett 1996; 21: 2023-2025.

6 Will M, Nolte S, Chichkov BN, Tüennermann A. Optical properties of waveguides fabricated in fused silica by femtosecond laser pulses. App/ Opt 2002; 41: 43604364.

7 Maruo S, Nakamura O, Kawata S. Three-dimensional microfabrication with twophoton-absorbed photopolymerization. Opt Lett 1997; 22: 132-134.

8 Sun HB, Matsuo S, Misawa H. Three-dimensional photonic crystal structures achieved with two-photon-absorption photopolymerization of resin. Appl Phys Lett 1999; 74: 786-788.

9 Cumpston BH, Ananthavel SP, Barlow S, Dyer DL, Ehrlich JE et al. Two-photon polymerization initiators for three-dimensional optical data storage and microfabrication. Nature 1999; 398: 51-54.

10 Kawata S, Sun HB, Tanaka T, Takada K. Finer features for functional microdevices. Nature 2001; 412: 697-698.

11 Serbin J, Ovsianikov A, Chichkov B. Fabrication of woodpile structures by two-photon polymerization and investigation of their optical properties. Opt Express 2004; 12 : 5221-5228.

12 Guo R, Xiao S, Zhai X, Li J, Xia A et al. Micro lens fabrication by means of femtosecond two photon photopolymerization. Opt Express 2006; 14: 810-816.

13 Reinhardt C, Passinger S, Chichkov BN, Marquart C, Radko IP et al. Laser-fabricated dielectric optical components for surface plasmon polaritons. Opt Lett 2006; 31 : 1307-1309.

14 Schlie S, Ngezahayo A, Ovsianikov A, Fabian T, Kolb HA et al. Three-dimensional cell growth on structures fabricated from ORMOCER $\mathrm{B}_{\circledast}$ by two-photon polymerization technique. J Biomater App/ 2007; 22: 275-287.

15 Ovsianikov A, Schlie S, Ngezahayo A, Haverich A, Chichkov BN. Two-photon polymerization technique for microfabrication of CAD-designed 3D scaffolds from commercially available photosensitive materials. J Tissue Eng Regen Med 2007; 1: 443-449.

16 Takada K, Sun HB, Kawata S. Improved spatial resolution and surface roughness in photopolymerization-based laser nanowriting. App/ Phys Lett 2006; 86: 071122.

17 Seet KK, Juodkazis S, Jarutis V, Misawa H. Feature-size reduction of photopolymerized structures by femtosecond optical curing of SU-8. Appl Phys Lett 2006; 89: 024106.

18 Haske W, Chen VW, Hales JM, Dong W, Barlow S et al. $65 \mathrm{~nm}$ feature sizes using visible wavelength 3-D multiphoton lithography. Opt Express 2007; 15: 3426-3436.

19 Tan D, Li Y, Qi F, Yang H, Gong Q. Reduction in feature size of two-photon polymerization using SCR500. Appl Phys Lett 2007; 90: 071106.

20 Emons M, Obata K, Binhammer T, Ovsianikov A, Chichkov BN et al. Two-photon polymerization technique with sub-50 nm resolution by sub-10 fs laser pulses. Opt Mater Express 2012; 2: 942-947.

21 Dip-In Laser Lithography: DILL (http://www.nanoscribe.de/de/technologie/dill).

22 Obata K, Koch J, Hinze U, Chichkov BN. Multi-focus two-photon polymerization technique based on individually controlled phase modulation. Opt Express 2010; 18: 17193-17200.

23 Gittard SD, Nguyen A, Obata K, Koroleva A, Narayan RJ et al. Fabrication of microscale medical devices by two-photon polymerization with multiple foci via a spatial light modulator. Biomed Opt Express 2011; 2: 3167-3178. 